\title{
Range finding of Alfvén oscillations and direction finding of ion-cyclotron waves by using the ground-based ULF finder
}

\author{
A. Guglielmi ${ }^{1}$, J. Kangas ${ }^{2}$, D. Milling ${ }^{3}$, D. Orr ${ }^{3}$, O. Pokhotelov ${ }^{1}$ \\ ${ }^{1}$ Institute of Physics of the Earth, 123810, Moscow, Russia \\ ${ }^{2}$ Department of Physical Sciences, University of Oulu, Linnanmaa, PO Box 333, FIN-90571 Oulu, Finland \\ ${ }^{3}$ University of York, Heslington, UK
}

Received: 13 May 1996 / Revised: 14 November 1996 / Accepted: 10 December 1996

\begin{abstract}
A new approach to the problem of direction and distance finding of magnetospheric ULF oscillations is described. It is based on additional information about the structure of geoelectromagnetic field at the Earth's surface which is contained in the known relations of the theory of magnetovariation and magnetotelluric sounding. This allows us to widen the range of diagnostic tools by using observations of Alfvén oscillations in the Pc 3-5 frequency band and the ion-cyclotron waves in the Pc 1 frequency band. Preliminary results of the remote sensing of the magnetosphere at low-latitudes using the MHD ranger technique are presented. The prospects for remote sensing of the plasmapause position are discussed.
\end{abstract}

\section{Introduction}

The study is devoted to the problem of direction and range finding of the ULF geoelectromagnetic oscillations. Our approach to this problem is influenced by the paper of Hayakawa (1991) dedicated to the direction finding of VLF/ELF emissions (see also Hayakawa et al., 1991). We describe the operation of the ULF finder as a device which makes it possible to complete the range finding of Pc 3-5 oscillations $(2-100 \mathrm{mHz})$ and the direction finding of Pc 1 waves $(0.2-5 \mathrm{~Hz})$. The range finder consists of a magnetometer and the recording system for measuring the Earth's currents, i.e. the normal set of standard observatory equipment. Moreover, we consider a large volume of the Earth's crust in the vicinity of the observation point as an essential element of the device. The dimensions of the volume are assumed to be of the order of the skin depth. The electrodynamic characteristics of the new element are

Correspondence to: J. Kangas taken into account when measurements and calculations are being carried out.

Figure 1 depicts a small part of the Earth's surface in the neighborhood of the observatory. Here the $x$ axis is directed northward, the $y$ axis points eastward and the horizontal line stands for the projection of a magnetic shell on the Earth's surface. We consider that this shell undergoes resonant Alfvén oscillations and our purpose is to locate this shell. We present our method which allows us to solve this problem by observations at one single point. In other words, we suggest an algorithm for measurements and calculations to determine the distance $x_{R}$ up to the projection of the oscillating magnetic shell on the ground.

The ULF finder is also available for the Pc 1 wave propagation, i.e., for the measurement of the angle $\varphi$ (see Fig. 1). We start with this problem as it is easier in some respects than the range finding of $\mathrm{Pc} 3-5$ oscillations. Although we cannot show any relevant Pc 1 data to verify the method at the moment, we introduce the basic elements of the direction finding method for the sake of completeness.

\section{Direction finding of Pc 1 pulsations}

It is customary to assume that Pc 1 are generated in the magnetosphere as ion-cyclotron waves, which propagate to the ionosphere in a longitudinal wave guide. Here a portion of wave energy is incident in the ionospheric wave guide and propagates horizontally along the Earth's surface at large distances from the end of the longitudinal wave guide (Manchester, 1966, 1968; Tepley and Landshoff, 1966; Greifinger and Greifinger, 1968, 1973). Measurement at one observation point thus tells us nothing about the location of this end. The problem has been solved by several methods such as triangulation direction of arrival (Frazer and Wawrzyniak, 1978), directional analysis (Chetaev, 1978), dispersion and group delay (see Feygin et al. 1979 and references therein) and by using the measurements from 


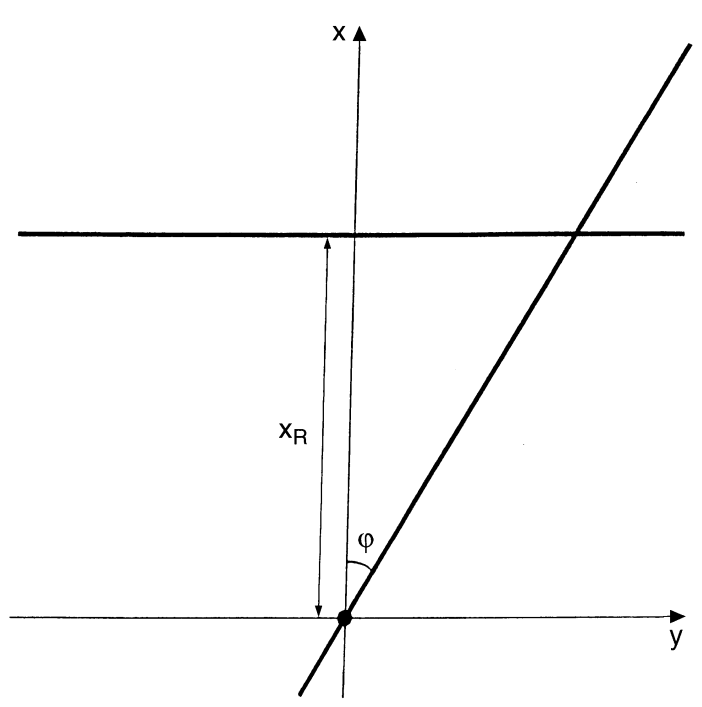

Fig. 1. The location of the ULF finder (point at the origin of the coordinates) relative to the projection of the oscillating magnetic shell onto the ground (horizontal line). The inclined line indicates the direction of wave propagation in the ionospheric wave guide

a dense network of magnetometers (Hayashi et al., 1982, 1988). In addition to these methods we present here an independent technique for the measurement of azimuth $\varphi$ of the Pc 1 propagation (see Fig. 1). The position of the Pc 1 source region may be established by the direction finding of Pc 1 at two or more points.

Let us locate the observation point in the center of a region with a relatively homogeneous distribution of conductivity of the rocks in horizontal directions. We shall register three components of the magnetic field $\mathbf{b}(t)$ in the Pc 1 frequency band. The data analysis is based on the following graphic construction.

We can determine the plane perpendicular to $\mathbf{b}(t)$. This plane intersects the horizontal plane (or Earth) in a straight line. The line rotates because the Pc 1 exhibits elliptic or, more precisely, quasi-elliptic polarization. The rotating line is coincident with the direction of $\mathrm{Pc} 1$ propagation twice over the period of oscillations, much as a stopped clock shows the right time twice a day. This leads to the question: when does this coincidence take place? The answer runs as follows: the rotating line coincides with the propagation direction when $b_{z}(t)=0$. Let us put this in another way. Figure 2 shows the hodograph of the vector-function $\mathbf{b}_{\tau}(t)$, where index $\tau$ indicates that we are dealing with the horizontal vector projection. The moments at which $b_{z}(t)=0$ are indicated by the bold dots on the hodograph. Let us draw a line which connects these two points. Then the perpendicular to this line defines the direction of Pc 1 propagation.

In order to prove this conclusion we make use of the relation

$b_{z}=i \lambda \varsigma \nabla_{\tau} \cdot \mathbf{b}_{\tau}$,

where $\varsigma$ stands for the surface impedance of the Earth, $\lambda=c / \omega, c$ is the velocity of light, $\omega$ is the frequency of

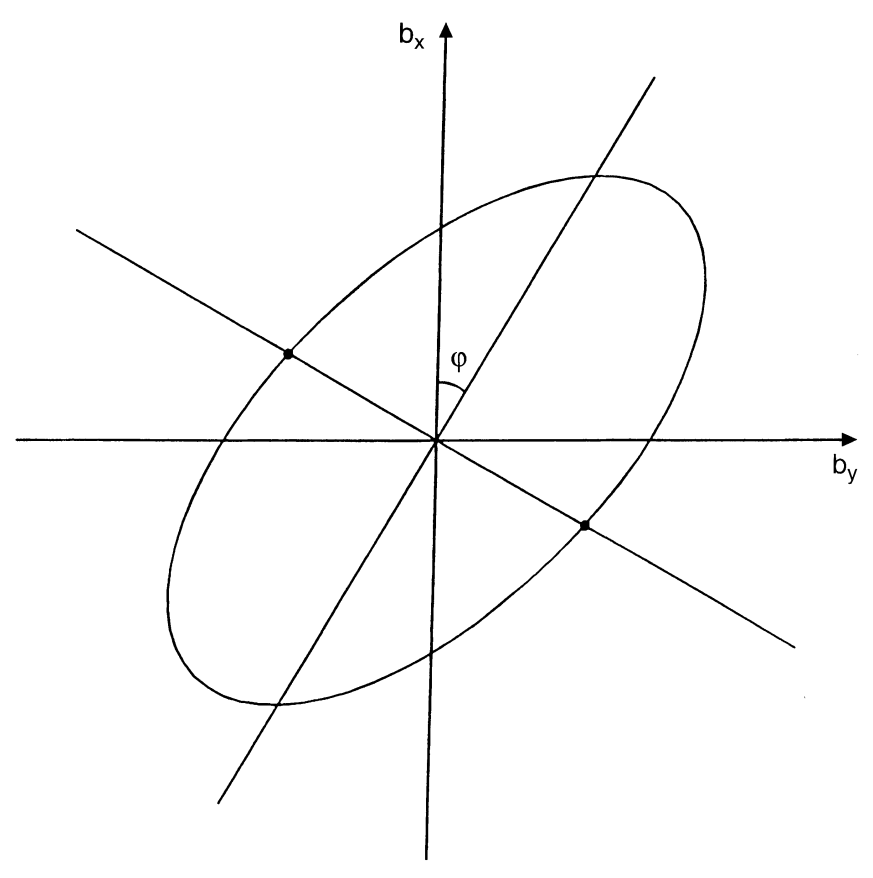

Fig. 2. The hodograph of vector-function $\mathbf{b}_{t=\tau}(t)$. The two points in the hodograph correspond to the moments at which $b_{z}(t)=0$

oscillations and $\mathbf{b}_{\tau}=\left(b_{x}, b_{y}\right)$. This relation is widely used in the study of the Earth's crust and upper mantle by means of magnetovariation sounding (Berdichevsky et al., 1969; Schmueker, 1970; Lilley and Sloane, 1976; Jones, 1980; Pajunpää, 1988).

Setting $b_{z}=0$, we obtain $\nabla_{\tau} \cdot \mathbf{b}_{\tau}=0$. The operator $\nabla_{\tau}$ may be replaced with $i \mathbf{k}_{\tau}$, where $\mathbf{k}_{\tau}=\left(k_{x}, k_{y}\right)$ is the local wave vector of horizontal propagation. Hence $\mathbf{b}_{\tau} \cdot \mathbf{k}_{\tau}=0$, i.e., vector $\mathbf{b}_{\tau}$ is parallel with respect to the wave front, and it is perpendicular to the direction of propagation when $b_{z}=0$.

The information on the surface impedance is not necessary when the assumption of horizontal homogeneity and isotropy of the Earth's crust in the vicinity of observation point is fulfilled. If it is necessary, we can take into account a weak dependence of $\varsigma$ on $x, y$. All one has to do is to replace $b_{z}$ by $b_{z}-i \lambda_{\mathbf{b}_{\tau}} \cdot \nabla \varsigma$. This replacement follows from the evident generalization of Eq. (1) to the case of slightly inhomogeneous media: $b_{z}=i \lambda \nabla_{\tau}\left(\varsigma_{\mathbf{b}_{\tau}}\right)$. The realization of this version requires preliminary investigation of the geoelectric structure of the region in the vicinity of the observation point to calculate $\nabla \zeta$. In this case the information on geoelectric properties of the rocks is used here in the explicit form.

Assuming the medium to be horizontally homogeneous, but anisotropic we can use the procedure described, replacing $\mathbf{b}_{\tau}$ by the auxiliary vector

$\beta_{i}=b_{j} \varsigma_{k k} \varsigma_{j i}^{-1}$,

where $s_{i j}$ stands for the surface impedance tensor and the indices $i, j, k$ take the values $x, y$. Here we consider that $\varsigma_{i j}=\varsigma_{j i}$. 


\section{MHD ranger}

We measure the east-west component of the electric field $E_{y}$ and the vertical component of the magnetic field $b_{z}$ in the Pc 3-5 frequency range. Let us make use of spectral analysis and designate $E=\left|E_{y}(\omega)\right|, Z=\left|b_{z}(\omega)\right|$. We may find the distance from the observation point to the magnetic shell, resonating at the frequency $\omega$, from the expression

$x_{R}(\omega)=\lambda(E / Z) \sin \theta$,

if the electroconductivity of rocks has horizontal homogeneous distribution (Guglielmi, 1989). Here $\theta(\omega)$ is the phase difference between the spectral components $E_{y}(\omega)$ and $b_{z}(\omega)$. In the case of inhomogeneous distribution it is necessary to replace $b_{z}$ by $b_{z}-i \lambda \mathbf{b}_{\tau} \cdot \nabla \varsigma$ as before.

Equation (2) was derived with the help of Eq. (1) and the impedance boundary condition

$E_{\tau}=\varsigma \mathbf{b}_{\tau} \times \mathbf{n}$,

where $\mathbf{n}$ denotes the unit vector normal to the Earth's surface directed earthwards (e.g., Wait, 1982), with regard to the approximate expression

$b_{x}(x)=b_{x}\left(x_{R}\right)\left[1-i\left(x-x_{R}\right) \Delta^{-1}\right]^{-1}$

for the known spatial structure of the field-line resonance (e.g., Hasegawa and Chen, 1974; Southwood, 1974; Orr and Hanson, 1981; Gough and Orr, 1984). Here $\Delta$ is the width of a resonance.

Let us draw an analogy between the range finding of field-line resonances based on Eq. (3) and magnetotelluric sounding (MTS). It is well known that the essence of MTS lies in the estimation of the vertical distribution of the crustal conductivity by the frequency dependence of the modulus of surface impedance (e.g., Rokityansky, 1982; Wait, 1982). The modulus of the surface impedance can be calculated from the Cagniard relation:

$|\varsigma|=E / H$.

Here $H=\left|b_{x}\right|$, see Eq. (3). Clearly MTS is based on the idea of compensation: since $E_{y}$ and $b_{x}$ are proportional to the intensity of external sources, their ratio $\left(E_{y} / b_{x}\right)$ does not depend on this intensity and moreover it is proportional to $\zeta$. (We recall that $E_{y}$ is proportional to $\zeta$, and $b_{x}$ mostly does not depend on $\zeta$.) In other words, MTS is related to the compensation of the indefinite conditions in the upper half-space. Similar to MTS, range finding in the form of Eq. (2) is achieved owing to a specific compensation: as both components $E_{y}$ and $b_{z}$ are proportional to $\zeta$, their ratio $\left(E_{y} / b_{z}\right)$ does not depend on $\zeta$. In other words, our method is directed to the compensation of indefinite conditions in the lower half-space.

\section{Verification}

As a test of the techniques described the data generously supplied by Prof. D. Chetaev has been analyzed in order to calculate $x_{R}$. Pc 3 pulsations have been observed in Ukraine not far from the village of Rakhny Sobovy,

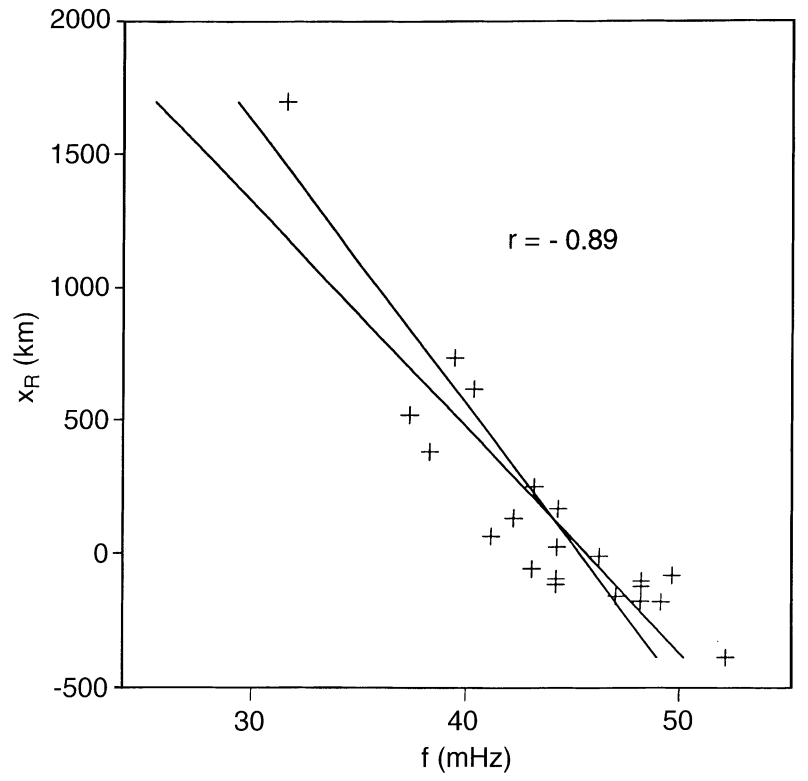

Fig. 3. Range finding at the latitude $43.9^{\circ}$ on August 30, 1974

$\phi_{0}=43.9^{\circ}$. Altogether 21 events suitable for analysis were registered on August 30, 1974 between 09.30-12.30 LT. Each event represents a wave packet with a carrier frequency $f=\omega / 2 \pi$ and a smoothly varying amplitude envelope. In addition to the distance $x_{R}$ also the impedance $|\zeta|$ has been calculated for selected events.

The dependence of $x_{R}$ on $f$ is shown in Fig. 3. We can see that the connection between $x_{R}$ and $f$ is obvious. The correlation coefficient $r=-0.89$ has the expected sign (the period $T=1 / f$ increases with increasing latitude). The regression equation is

$x_{R}=3.76 \times 10^{3}-82.7 f$,

where $x_{R}$ is measured in $\mathrm{km}$ and $f$ in $\mathrm{mHz}$, respectively.

At the same time the connection between $|\zeta|$ and $f$ turned out to be rather weak (see Fig. 4). The correlation coefficient $r=0.24$. This indicates that, at least in the case under study, the method of the magnetospheric MHD-range finding is more informative than that of magnetotelluric sounding.

The result obtained may be verified, for instance, by an observation in two points displaced in latitude. As such data is not available, we carried out indirect verification by means of analysis of pulsation polarization in the horizontal plane. Figure 5 displays the relative distributions of number of cases versus $x_{R}$ for $L$ and $R$ polarizations separately. One can see that $L$ polarization is preferable if $x_{R}>0$ and vice versa as would be expected, in the framework of the theory of field-line resonances (e.g., Lanzerotti, 1976).

With a knowledge of $x_{R}$ and the position of the device, it is possible to determine the magnetic shell $L$ value. Also, knowing $L$ and the frequency of resonant oscillations, we can use the familiar method of hydromagnetic diagnostics (HMD) for the estimation of plasma density $\rho$ in the equatorial plane of the 


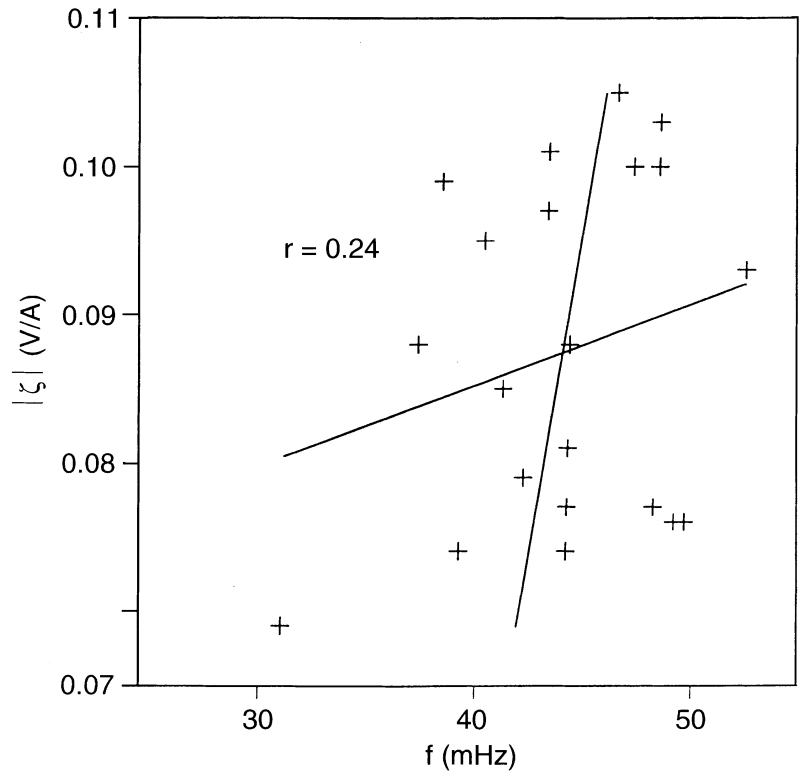

Fig. 4. Magnetotelluric sounding at the same latitude on the same date

magnetosphere (e.g., Aubry, 1970; Jacobs, 1970; Orr, 1973; Orr and Matthew, 1971; Nishida, 1978). According to Eq. (6) $d \lg \rho / d L \simeq-0.9$ at $L \simeq 2$, which is not in contradiction with direct and indirect measurements (e.g., Carpenter and Anderson, 1992).

\section{Discussion}

We now discuss mainly two questions, one related to MTS and the other concerns HMD, and in one way or another both of these questions are related to the

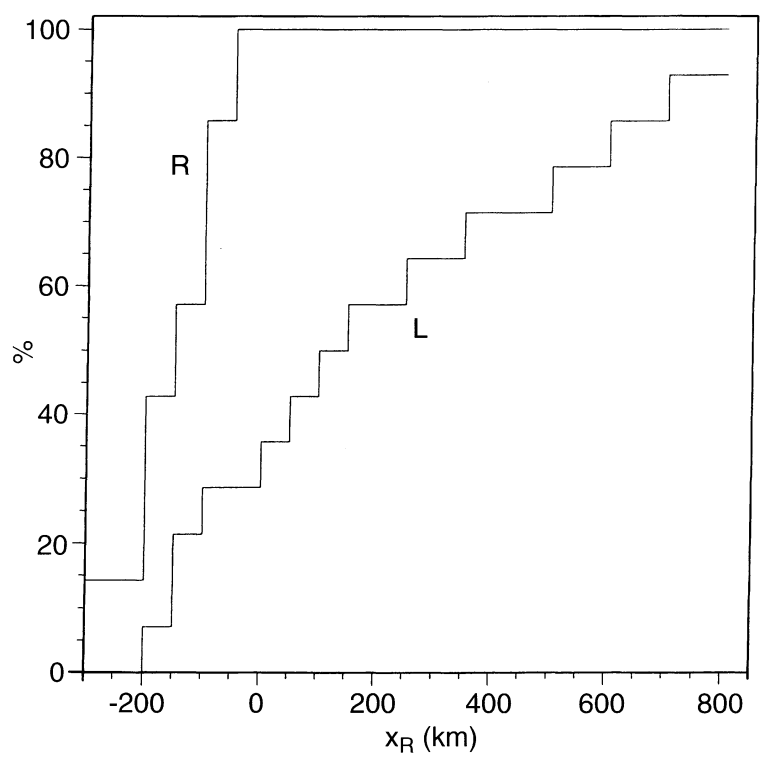

Fig. 5. Distribution of the polarization of pulsations with latitude problem of ULF finding. We point out also some limitations in using the direction finding method.

In order to understand the reason why the impedance $\varsigma$ is almost independent of the frequency $f$ (Fig. 4), let us consider the relation

$E_{y}=-\varsigma\left(b_{x}+\frac{i \delta^{2}}{4} \frac{\partial^{2} b_{x}}{\partial x^{2}}\right)$,

which is more general than the Cagniard relation Eq. (5). Here $\delta=\sqrt{2}|\varsigma| \lambda$ is the effective skin depth (e.g. Wait, 1982).

The strong inequality

$\frac{\delta^{2}}{4}\left|\frac{\partial^{2} b_{x}}{\partial x^{2}}\right| \ll\left|b_{x}\right|$

is usually valid, and that is why the second term on the right hand side of Eq. (7) may be omitted. Such a rejection leads to the violation of the correlation between $\varsigma$ and $f$. Let us consider the question in a greater detail.

As we may write

$\frac{\partial^{2} b_{x}}{\partial x^{2}}=\frac{2 b_{x}}{\left(\Delta+i x_{R}\right)^{2}}$,

it follows from Eqs. (1) and (4) that $\left(\Delta+i x_{R}\right)=$ $-\lambda \varsigma b_{x} / b_{z}$ at $x=0$. Based on these relations we conclude that the rejection of the second term in the right hand side of Eq. (7) leads to an error of the order of $\sim(Z / H)^{2}$. The analysis of data indicates that the random variation of value $(Z / H)^{2}$. The analysis of data indicates that the random variation of value $(Z / H)^{2}$ is of the order of $5-10 \%$.

On the other hand, the relative variation of frequency is of the order of $10 \%$ (see Fig. 3). It is known that $\varsigma$ depends on frequency such as $\sqrt{f}$ in the high frequency limit, and $\varsigma$ is almost independent on $f$ in the low frequency limit. Hence, the regular variation of $|\varsigma|$ in our case is not greater than $5 \%$, i.e., it is less than a random variation. Therefore, the poor correlation between $|\varsigma|$ and $f$ is the result of neglecting the second term on the right hand side of Eq. (7).

A more detailed analysis of the data available for the present study reinforces our conclusion. It has been found that the correlation coefficient $r$ tends to increase with the increase of distance $\left|x_{R}\right|$. For example, $r=0.41$ if $\left|x_{R}\right|>100 \mathrm{~km}$ (14 events), and $r=0.07$ if $\left|x_{R}\right|<200 \mathrm{~km}$ (14 events). The reason is that the greater $\left|x_{R}\right|$, the smaller the second term in the right hand side of Eq. (7).

We now turn our attention to the discussion of the application of ULF range finding to HMD. We see great possibilities for the development of hydromagnetic diagnostics with the help of the MHD-ranger. The idea is as follows. If the range-finder is placed in the band of latitude $50-60^{\circ}$ it may be possible to detect the plasmapause position $L$ by recording $\mathrm{Pc} 3,4$ pulsations. It is anticipated that a display of $x_{R}$ against $f$ will reveal the location of the plasmapause by a distinct discontinuity.

There are two weak points which have to be recognized when applying the direction finding method 
developed in Sect. 2. Although Hayashi et al. (1982) found that the radius of the ionospheric secondary source of Pc 1 pulsations does not exceed $300 \mathrm{~km}$ it is obvious that the $\mathrm{Pc} 1$ source region is often more extended in the E-W direction. On the other hand, according to the theoretical calculations (Greifinger and Greifinger, 1973; Fujita, 1987) the propagation of Pc 1 waves is limited mostly along the magnetic meridian plane i.e., at small values of $\varphi$. Although this may not be the general case according to our experience (see also e.g., Fraser, 1975) it is probable that the method to locate the Pc 1 source by the direction finding method does not work in all cases. However, it is expected that the method always produces useful information about the fluctuations of the local direction of the Pc 1 propagation.

\section{Conclusion}

The basic idea of the present study is that the impedance relationships between the components of electromagnetic field on the Earth's surface can be used in hydromagnetic diagnostics. Two simple methods based on the impedance relation (1) have been introduced. This is not, however, an exact formula. We have avoided discussion of the applicability conditions but even so it is clear that the depth of field penetration into the crust must be small as compared with the vacuum wavelength, the radius of curvature of the surface and the characteristic scales of the changes in the field and medium properties along the surface of the Earth. Any complete answer to the question of applicability condition is difficult to present. The relationship (1) depends strongly on the geoelectric conditions in the vicinity of the point of observation. There is always the risk that one or another applicability condition will be violated or, alternatively, that some condition will turn out to be too rigid.

Is it possible to suggest an alternative approach which would retain the idea in general terms but which would not be based directly on Eq. (1)? We believe that the answer is positive. For this purpose we need to study the conductivity distribution in the lower half-space near the observation point by MTS methods and then jointly solve the internal problem (for the Earth's crust) and the external problem (for the magnetosphere). The solutions are to meet at the interface. Then, it becomes possible to use additional relationships between the field components in order to improve the accuracy of magnetospheric diagnostics.

Acknowledgments. This research was partly supported by the Commission of the European Union (Brussels) through Research Grant INTAS-94-2811. The authors are grateful to Professor Dmitrii Chetaev for the kind permission to use his data. We acknowledge also the stimulating comments and criticism of the referees.

Topical Editor K.-H. Glaßmeier thanks three referees for their help in evaluating this paper.

\section{References}

Aubry, M. P., Diagnostics of the magnetosphere from ground based measurements of electromagnetic waves, Ann. Geophysicae, 26, 341, 1970.

Berdichevsky, M. N., L. L. Vanyan, and E. B. Fainberg, Magnetic variation sounding using the space derivatives of field, Geomagn. Aeronom., 9, 229-301, 1969.

Carpenter, D. L., and R. R. Anderson, An ISEE/whistler model of equatorial density in the magnetosphere, J. Geophys. Res., A97, 1097-1108, 1992.

Chetaev, D. N., On the local structure of magnetotelluric field, $I z v$. AN SSSR, Ser. Fiz. Zemli, 10, 105-116, 1978.

Feygin, F. Z., Y. P. Kurchashov, V. A. Troitskaya, D. S. Fligel, and K. Dobes, Method of locating the Pc 1 source and hot plasma parameters in the generation region, Planet. Space Sci., 27, 151$158,1979$.

Fraser, B. J., Ionospheric duct propagation and Pc 1 pulsation sources, J. Geophys. Res., 80, 2790-2796, 1975.

Fraser, B. J., and S. S. Wawrzyniak, Source movements associated with IPDP pulsations, J. Atmos. Terr. Phys., 40, 1281-1288, 1978.

Fujita, S., Duct propagation of a short-period hydromagnetic wave based on the international reference ionosphere model, Planet, Space Sci., 35, 91-103, 1987.

Gough, H., and D. Orr, The effect of damping on geomagnetic pulsation amplitude and phase at ground observatories, Planet. Space Sci., 32, 619-628, 1984.

Greifinger, C., and P. S. Greifinger, Theory of hydromagnetic propagation in the ionospheric wave guide, J. Geophys. Res., 73, 7473-7490, 1968.

Greifinger, C., and P. Greifinger, Wave guide propagation of micropulsations out of the plane of the geomagnetic meridian, J. Geophys. Res., 78, 4611-4618, 1973.

Guglielmi, A., Diagnostics of the plasma in the magnetosphere by means of measurement of the spectrum of Alfvén oscillations, Planet, Space Sci., 37, 1011-1012, 1989.

Hasegawa, A., and L. Chen, Theory of magnetic pulsations, Space Sci. Rev., 16, 347-359, 1974

Hayakawa M., Direction finding of magnetospheric VLF/ELF emissions and their generation mechanism in Environmental and Space Electromagnetics, Ed. H. Kikuchi, Springer, Tokyo Berlin Heidelberg, 155-167, 1991.

Hayakawa M., K. Ohta, and S. Shimakura, Direction finding of very-low-latitude whistlers and their propagation in Environmental and Space Electromagnetics, Ed. H. Kikuchi, Springer, Tokyo Berlin Heidelberg, 168-171, 1991.

Hayashi, K., S. Kokubun, T. Oguti, K. Truruda, S. Mashida, T. Kitamura, O. Saka, and T. Watanabe, The extent of Pc 1 source region in high latitudes, Can. J. Phys., 59, 1097-1105, 1982.

Hayashi, K., T. Yamamoto, S. Kukubun, T. Oguti, T. Ogawa, N. Iwagami, T. Araki, T.-I. Kitamura, O. Saka, K. Makita, N. Sato, T. Watanabe, R. E. Horita, D. J. McEwen, J. S. Kim, and A. Egeland, Multi-station observation of Ipdp micropulsations-two-dimensional distribution and evolution of the source region, J. Geomag. Geoelectr., 40, 583-619, 1988.

Jacobs, J. A., Geomagnetic micropulsations, Springer, New York Berlin Heidelberg, 1970.

Jones, A. G., Geomagnetic induction studies in Scandinavia, J. Geophys., 48, 181-194, 1980.

Lanzerotti, L. J., Hydromagnetic waves: International symposium on solar-terrestrial physics, Boulder, Colorado, June 7-18, 1976.

Lilley, F. E. M., and M. N. Sloance, On estimation electrical conductivity using gradient data from magnetometer arrays, J. Geomagn. Geoelectr., 28, 321-328, 1976.

Manchester, R. N., Propagation of Pc 1 micropulsations from high to low latitudes, J. Geophys. Res., 71, 3749-3754, 1966. 
Manchester, R. N., Correlation of Pc 1 micropulsations at spaced stations, J. Geophys. Res., 73, 3549-3556, 1968.

Nishida, A., Geomagnetic diagnosis of the magnetosphere, Springer, New York Berlin Heidelberg, 1978.

Orr, D., Magnetic pulsations within the magnetosphere: a review, J. Atmos. Terr. Phys., 35, 1-50, 1973.

Orr, D., and J. A. D. Matthew, The variation of geomagnetic micropulsation period with latitude and plasmapause, Planet. Space Sci., 19, 897-905, 1971.

Orr, D., and H. W. Hanson, Geomagnetic pulsation phase patterns over an extended latitude array, J. Atmos. Terr. Phys., 43, 894 910, 1981.

Pajunpää, K., Application of the horizontal spatial gradient technique to magnetometer array data in Finland, Rep. Dept. Geophys. Univ. Oulu, Oulu, Finland, 15, 1-14, 1988.
Rokityansky, I. I., Geoelectric investigation of the Earth's crust and mantle, Springer, Berlin Heidelberg New York, 1982.

Schmueker, U., An introduction to induction anomalies, J. Geomagn. Geoelectr., 22, 9, 1970.

Southwood, D. J., Recent studies in micropulsation theory, Space Sci. Rev., 16, 413-425, 1974.

Tepley, L., and R. K. Landshoff, Wave guide theory for ionospheric propagation of hydromagnetic emissions, J. Geophys. Res., 71, 1499-1503, 1966.

Wait, J. R., Geo-Electromagnetism, Academic Press, New York, 1982. 